
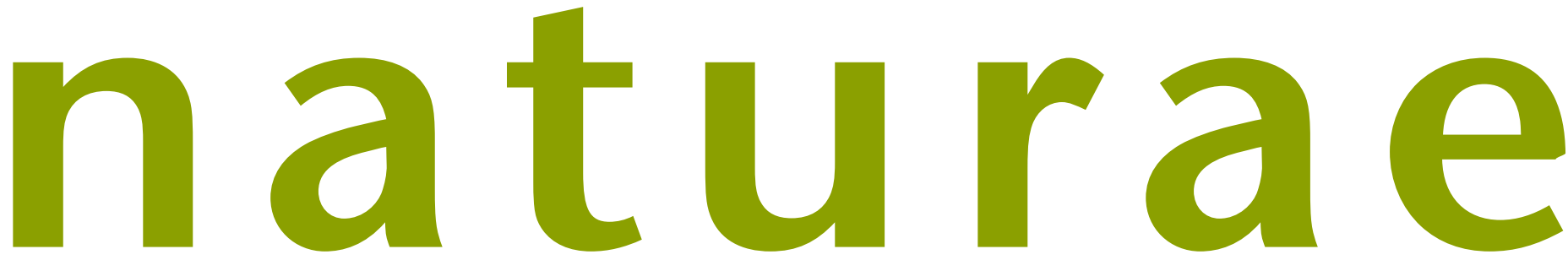

$2020 \cdot 6$

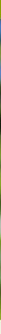

Quelle est la véritable surface du domaine vital des Tortues d'Hermann (Testudo hermanni Gmelin, 1789)? Implications pour la conservation

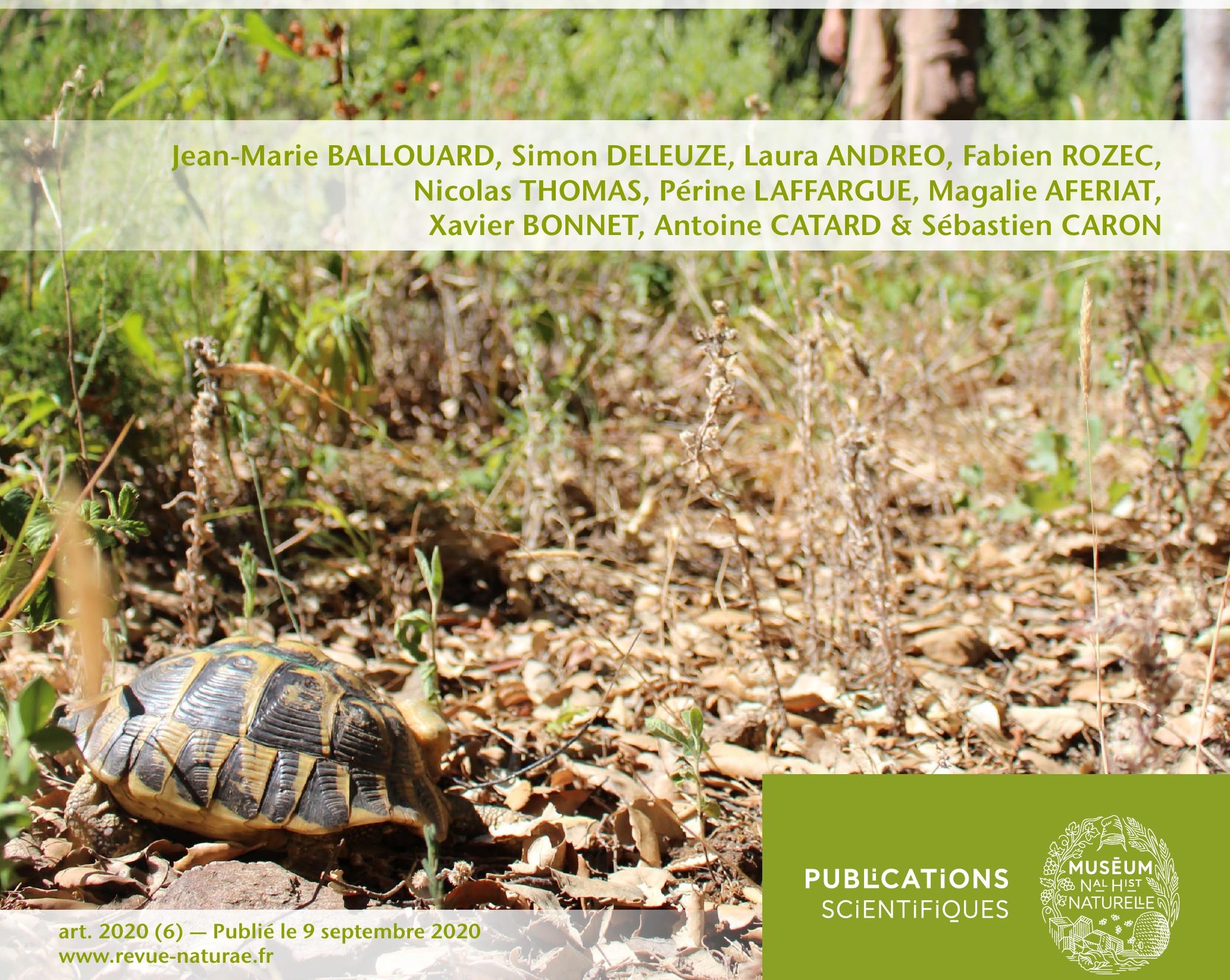


DiRECTEUR DE LA PUBLICATION / PUBLICATION DIRECTOR: Bruno David,

Président du Muséum national d'Histoire naturelle

RÉDACTEUR EN CHEF / EDITOR-IN-CHIEF: Jean-Philippe Siblet

ASSISTANTE DE RÉDACTION / ASSISTANT EDITOR: Sarah Figuet (naturae@mnhn.fr)

Mise EN PAGE / PAGE LAYOUT: Sarah Figuet

COMITÉ SCIENTIFIQUE / SCIENTIFIC BOARD:

Luc Abbadie (UPMC, Paris)

Luc Barbier (Parc naturel régional des caps et marais d'Opale, Colembert)

Aurélien Besnard (CEFE, Montpellier)

Vincent Boullet (Expert indépendant flore/végétation, Frugières-le-Pin)

Hervé Brustel (École d'ingénieurs de Purpan, Toulouse)

Patrick De Wever (MNHN, Paris)

Thierry Dutoit (UMR CNRS IMBE, Avignon)

Éric Feunteun (MNHN, Dinard)

Romain Garrouste (MNHN, Paris)

Grégoire Gautier (DRAAF Occitanie, Toulouse)

Olivier Gilg (Réserves naturelles de France, Dijon)

Frédéric Gosselin (Irstea, Nogent-sur-Vernisson)

Patrick Haffner (UMS PatriNat, Paris)

Frédéric Hendoux (MNHN, Paris)

Xavier Houard (OPIE, Guyancourt)

Isabelle Leviol (MNHN, Concarneau)

Francis Meunier (Conservatoire d'espaces naturels - Picardie, Amiens)

Serge Muller (MNHN, Paris)

Francis Olivereau (DREAL Centre, Orléans)

Laurent Poncet (UMS PatriNat, Paris)

Nicolas Poulet (AFB, Vincennes)

Jean-Philippe Siblet (UMS PatriNat, Paris)

Laurent Tillon (ONF, Paris)

Julien Touroult (UMS PatriNat, Paris)

COUVERTURE / COVER:

Tortue d'Hermann adulte (Testudo hermanni Gmelin, 1789) suivie à l'aide d'un radio-émetteur collé à l'arrière de sa carapace. Crédit photo: F. Pille.

Naturae est une revue en flux continu publiée par les Publications scientifiques du Muséum, Paris

Naturae is a fast track journal published by the Museum Science Press, Paris

Les Publications scientifiques du Muséum publient aussi / The Museum Science Press also publish:

Adansonia, Zoosystema, Anthropozoologica, European Journal of Taxonomy, Geodiversitas, Cryptogamie sous-sections Algologie, Bryologie, Mycologie, Comptes Rendus Palevol.

Diffusion - Publications scientifiques Muséum national d'Histoire naturelle

CP $41-57$ rue Cuvier F-75231 Paris cedex 05 (France)

Tél.: 33 (0)1 40794805 / Fax: 33 (0)1 40793840

diff.pub@mnhn.fr / http://sciencepress.mnhn.fr

(C) Publications scientifiques du Muséum national d'Histoire naturelle, Paris, 2020

ISSN (électronique / electronic): 1638-9387 


\section{Quelle est la véritable surface du domaine vital des Tortues d'Hermann (Testudo hermanni Gmelin, 1789)? Implications pour la conservation}

Jean-Marie BALLOUARD

Simon DELEUZE

Laura ANDREO

Station d'Observation et de Protection des Tortues et de leurs Milieux, Centre de Recherche et de Conservation des Chéloniens (SOPTOM-CRCC), 1065, Route du Luc, F-83660 Carnoules (France) jean-marie.ballouard@soptom.org simon.deleuze@gmail.com laura.andreo13@gmail.com

Fabien ROZEC

Nicolas THOMAS

Communauté d'Agglomération Var Estérel Méditerranée - CAVEM 624 Chemin Aurélien, F-83700 Saint-Raphaël (France) f.rozec@cavem.fr n.thomas@cavem.fr

Périne LAFFARGUE

Conservatoire d'Espaces naturels Provence-Alpes-Côte d'Azur Pôle Var, L'Astragale, 888 chemin des Costettes, F-83340 Le Cannet des Maures (France) perrine.laffargue@cen-paca.org

Magalie AFERIAT

Station d'Observation et de Protection des Tortues et de leurs Milieux, Centre de Recherche et de Conservation des Chéloniens (SOPTOM-CRCC),

1065, Route du Luc, F-83660 Carnoules (France) mag.aferiat@orange.fr

Xavier BONNET Centre d'Étude Biologique de Chizé, UMR-7372, CNRS-Université de La Rochelle, F-79360 Villiers en Bois (France) bonnet@cebc.cnrs.fr

Antoine CATARD Conservatoire d'Espaces naturels Provence-Alpes-Côte d'Azur Pôle Var, L'Astragale, 888 chemin des Costettes, F-83340 Le Cannet des Maures (France) antoine.catard@cen-paca.org

Sébastien CARON

Station d'Observation et de Protection des Tortues et de leurs Milieux, Centre de Recherche et de Conservation des Chéloniens (SOPTOM-CRCC), 1065, Route du Luc, F-83660 Carnoules (France) sebastien.caron@soptom.org 
MOTS CLÉS Milieu méditerranéen, Reptile,

radio-tracking polygone convexe minimum aire de protection.

\footnotetext{
KEY WORDS

Mediterranean habitat, reptile,

radio-tracking,

minimum convex polygon,

protected areas.
}

Ballouard J.-M., Deleuze S., Andreo L., Rozec F., Thomas N., Laffargue P., Aferiat M., Bonnet X., Catard A. \& Caron S. 2020. - Quelle est la véritable surface du domaine vital des Tortues d'hermann (Testudo hermanni Gmelin, 1789)? Implications pour la conservation. Naturae 2020 (6): 101-111. https://doi.org/10.5852/naturae2020a6

\section{RÉSUMÉ}

L'estimation précise de la taille des domaines vitaux (DV) qu'occupent les individus d'une espèce est cruciale pour les gestionnaires. Elle permet de mieux définir la taille minimale des sites à protéger et de limiter la fragmentation des populations. Grâce à des suivis par radio-tracking menés sur l'ensemble de la saison d'activité, les domaines vitaux annuels de 165 Tortues d'Hermann (Testudo hermanni Gmelin, 1789) provenant de neuf sous-populations de France continentale ont été obtenus par la méthode des polygones convexes minimum (MCP). Les grands domaines vitaux mesurés sur plusieurs populations contrastent avec les données précédemment publiées sur le sujet. Notre étude montre que le DV d'une Tortue d'Hermann occupe environ neuf hectares (valeur médiane 5,2 ha) alors que la valeur moyenne de référence pour les populations françaises n'est que de deux hectares. Cette différence s'explique par les petites tailles d'échantillons obtenus sur de courtes périodes des études précédentes. Les suivis intensifs menés pendant 10 ans fournissent une vision plus réaliste de la taille des domaines vitaux. En accord avec la littérature, une grande variabilité interindividuelle se traduit par des valeurs extrêmes, éloignées des DV (0,6 ha à 95,0 ha). Globalement, la taille des DV augmente au printemps lorsque l'activité des individus est la plus importante et se stabilise pendant la saison estivale, voire en automne. Il est essentiel que l'établissement de zones de protection et de corridors prenne en compte ces nouvelles données, tout particulièrement les valeurs maximales ( $>15$ ha). Seules les populations suffisamment grandes occupant des habitats diversifiés pourront s'adapter aux changements globaux et aux changements climatiques notamment. Protéger de grandes surfaces si possible connectées par des infrastructures dédiées (e.g. passages sous les routes) est une condition pour avoir une diversité génétique importante et une diversité de micro-habitats suffisante.

\begin{abstract}
What is the real home range of the Hermann's Tortoises (Testudo hermanni Gmelin, 1789)? Implication for conservation.

Accurate evaluation of animal home range is crucial for field managers. It provides key information to determine the surface of areas that require protection and to limit population fragmentation. Home ranges (MCP) were obtained in 165 Hermann tortoises radio-tracked across the whole activity season in nine populations of continental France. Our study shows that mean home range was considerably larger compared to previous estimates: 9ha (median 5.2ha) versus $2 \mathrm{ha}$. This strong difference is explained by the small sample sizes collected during short time periods in previous studies. Our intensive monitoring during 10 years provides more realistic estimates. In agreement with the literature, we observed wide inter-individual variations (0.6ha to 95.0ha). Home range size increased in spring, during the peak of activity, and tended to stabilize in summer or in autumn. It is essential to take into account realistic home range estimates, especially maximal values ( $>15 \mathrm{ha}$ ), to establish suitable protected areas and to create corridors among them. Large populations occupying large areas with diverse habitats are more likely to adapt global and climatic changes. Protecting large surfaces (e.g. hundreds of hectares) connected by dedicated infrastructures (under road passages) is recommended to maintain a high genetic diversity and to provide a wide range of microhabitats.
\end{abstract}

\section{INTRODUCTION}

Le domaine vital est l'aire qui contient les habitats et les ressources dont un individu a besoin depuis la naissance et durant toute sa vie (Burt 1943; White \& Garrot 1990). En général, la taille des domaines vitaux est influencée par la qualité des habitats (Gese et al. 1988; Hailey \& Coulson 1996; Castellón et al. 2018). D'autres paramètres sont importants, comme le sexe et l'âge des individus (Lagarde et al. 2003; Harless et al. 2010), ou encore la densité de population qui conditionne la probabilité de rencontre entre partenaires (Mazzotti et al. 2002), la présence d'obstacles, le climat et la topographie
(Guisan \& Hofer 2003; Franks et al. 2011). L'ensemble des domaines vitaux des individus délimite l'espace qui accueille une population (Powel \& Mitchell 2012). En conséquence, il est essentiel de connaître la taille et la forme des domaines vitaux d'un nombre suffisant d'individus pour assurer la protection des espèces (Börger et al. 2006; Kingsburry \& Robinson 2016). En effet, sans cette connaissance il est très difficile de définir les contours des aires à protéger (Maxwell et al. 2011; Di Franco et al. 2018) ou d'éviter la fragmentation des populations (Devillard et al. 2008; Hinam \& Clair 2008). Chez les organismes avec de faibles capacités de mouvement et donc de dispersion, comme les tortues terrestres, l'estimation 
TABLEAU 1. - Taille des domaines vitaux (en hectares) moyens disponible dans la littérature pour des Tortues d'Hermann Testudo hermanni Gmelin, 1789 (TH) et Testudo hermanni boetgerri Mojsisovics, 1889 (TB) parfois accompagnée selon les données disponibles avec l'erreur standard ( \pm ) ou les valeurs minimales et maximales entre crochets, selon la technique de suivi des individus (CMR, Capture Marquage Recapture; RT, Radiotracking), le nombre d'individus suivi (N), la durée du suivi et la méthode de calcul. *, indique si l'étude révèle une différence significative entre les sexes; MCP, Polygones Convexes Minimum; NA, absence d'information sur le sujet.

\begin{tabular}{|c|c|c|c|c|c|c|c|c|c|}
\hline Espèce & Pays & Population/Site & $\begin{array}{l}\text { Méthode } \\
\text { de suivi }\end{array}$ & $\mathbf{N}$ & $\begin{array}{l}\text { Durée } \\
\text { du suivi }\end{array}$ & $\begin{array}{l}\text { Méthode de } \\
\text { calcul }\end{array}$ & $\begin{array}{c}\text { Domaines } \\
\text { vitaux } \\
\text { (mâles) }\end{array}$ & $\begin{array}{l}\text { Domaines } \\
\text { vitaux } \\
\text { (femelles) }\end{array}$ & Références \\
\hline $\mathrm{TH}$ & France & Les Mayons & $\mathrm{CMR}+\mathrm{RT}$ & 7 & 1 & $\mathrm{MCP}$ & $2,4^{*}$ & 1,6 & $\begin{array}{l}\text { Swingland et al. } \\
1986\end{array}$ \\
\hline $\mathrm{TH}$ & France & La Môle & RT & 10 & NA & MCP & $0,9^{*}$ & 2,2 & $\begin{array}{l}\text { Huot- } \\
\text { Daubremont } \\
1996\end{array}$ \\
\hline $\mathrm{TH}$ & France & Vidauban & thread trailing & 18 & 1 mois & MCP & $1,2 \pm 1,1$ & $2,1 \pm-2$ & $\begin{array}{l}\text { Longepierre et al. } \\
2001\end{array}$ \\
\hline $\mathrm{TH}$ & Italie & $\begin{array}{c}\text { Bosco della } \\
\text { Mesola }\end{array}$ & RT & 8 & 6 mois & MCP & $\begin{array}{c}4,6^{\star} \\
{[4,4-8]}\end{array}$ & $\begin{array}{c}7,4 \\
{[1,6-10,8]}\end{array}$ & $\begin{array}{l}\text { Mazzotti et al. } \\
2002\end{array}$ \\
\hline $\mathrm{TH}$ & Italie & $\begin{array}{l}\text { Northern } \\
\text { Western Italy }\end{array}$ & RT & NA & 1 an & MCP & $0,7^{\star}$ & 1,5 & $\begin{array}{l}\text { Calzolai \& } \\
\quad \text { Chelazzi } 1991\end{array}$ \\
\hline $\mathrm{TH}$ & Italie & $\begin{array}{l}\text { Toscane } \\
\text { (Maremme } \\
\text { Natural Park) }\end{array}$ & NA & NA & 1 an & NA & {$[1,73-3,25]^{\star}$} & {$[0,89-4,17]$} & $\begin{array}{l}\text { Bossuto et al. } \\
2000\end{array}$ \\
\hline $\mathrm{TH}$ & Italie & $\begin{array}{l}\text { Monti Nebrodi } \\
\text { (Sicile) }\end{array}$ & NA & NA & NA & NA & {$[0,24-0,74]^{\star}$} & {$[0,27-68]$} & Tomasetti 1997 \\
\hline $\mathrm{TH}$ & Italie & PNR Marturanum & RT & 4 & 3-4 mois & MCP & 2,7 & 1,5 & $\begin{array}{l}\text { Luiselli et al. } \\
\quad 2009\end{array}$ \\
\hline $\mathrm{TH}$ & Espagne & Delta de I'Ebre & RT & 12 & 1 an & MCP & $2,7^{\star}$ & 1,8 & Bertolero 2002 \\
\hline $\mathrm{TH}$ & Espagne & Albert & RT & 13 & 14 mois & MCP & 2,6 & 3,2 & $\begin{array}{l}\text { Vilardell Batino } \\
\text { et al. } 2015\end{array}$ \\
\hline $\mathrm{TH}$ & Espagne & Albert & RT & 21 & 6 mois & Kernel 95 & $5,7 \pm 3,4^{*}$ & $3,24 \pm 2,36$ & $\begin{array}{r}\text { Casamitjana } \\
\text { et al. } 2012\end{array}$ \\
\hline TB & Serbie & Nis & CMR & & 3 ans & MCP & $\begin{array}{c}4,56 \\
{[n a-27]}\end{array}$ & $\begin{array}{c}7,53 \\
{[n a-90]}\end{array}$ & $\begin{array}{l}\text { Stojadinović et al. } \\
2017\end{array}$ \\
\hline TB & Roumanie & $\begin{array}{l}\text { Iron Gates Natural } \\
\text { Park }\end{array}$ & RT & 24 & 1 an & $\begin{array}{c}\text { MCP-Kernel } \\
\text { adjust }\end{array}$ & $3,02 \pm 1,08^{*}$ & $4,25 \pm 0,74$ & $\begin{array}{l}\text { Rozylowicz \& } \\
\text { Popescu } 2013\end{array}$ \\
\hline TB & Turquie & Kozyörük Village & $\mathrm{RT}+\mathrm{GPS}$ & 6 & 3 mois & MCP & 3,3 & - & $\begin{array}{l}\text { Türkozan et al. } \\
2019\end{array}$ \\
\hline
\end{tabular}

correcte de la taille des domaines vitaux est cruciale pour la gestion des milieux et des habitats.

La Tortue d'Hermann (Testudo hermanni Gmelin, 1789) est considérée comme l'une des espèces de reptiles les plus menacées en Europe occidentale (Corbett 1989). La sous-espèce Testudo hermanni hermanni Gmelin, 1789 présente en Europe de l'ouest est classée vulnérable par l' Union internationale pour la Conservation de la Nature (UICN) à l'échelle de la France métropolitaine (UICN France et al. 2015). Autrefois largement distribuées sur l'ensemble du bassin méditerranéen, les populations sont aujourd'hui limitées au sud-est du territoire français (le Massif des Maures, la Plaine des Maures et l'Estérel) ainsi qu'à la Corse, l'Italie et l'Espagne (Cheylan et al. 2009). L'espèce a fortement décliné, principalement à cause de la disparition et de la dégradation des habitats (Cheylan 1984; Livoreil 2009; Celse et al. 2018). Son statut d'espèce emblématique du bassin méditerranéen a justifié la création en 2009 de la réserve naturelle nationale de la Plaine des Maures $(5,276$ hectares) qui protège une vaste zone de la convoitise des aménageurs. Cet exemple montre que cette tortue peut servir d'espèce parapluie (Berardo et al. 2015).

Lespèce est phylopatrique, ce qui facilite la caractérisation des domaines vitaux (Chelazzi \& Francisci 1979). La littérature fait état de domaines vitaux moyens d'un à deux hectares en
France, en Italie et en Grèce (Swingland et al. 1986; Hailey 1989; Calzolai \& Chelazzi 1991 ; Bertolero et al. 2011; Vilardell-Bartino et al. 2015). L'espèce occupe des habitats variés allant du maquis ouvert à un habitat semi-forestier en passant par des zones de mosaïques agricoles ou de dunes littorales (Bertolero et al. 2011). Cette diversité d'habitats est susceptible d'influencer la taille des domaines vitaux (Bertolero et al. 2011).

Les suivis par radio-tracking sont particulièrement utiles pour mesurer de façon précise les domaines vitaux et les mouvements des individus (McMaster \& Downs 2009; Harless et al. 2010). Néanmoins, ils nécessitent un investissement logistique important qui contraint la durée des suivis. Pour qu' ils soient efficaces, notamment lorsqu'il s'agit de délimiter des zones de protection, les domaines vitaux doivent représenter l'ensemble des habitats utilisés par les individus au cours du cycle annuel. C'est le cas pour la Tortue d'Hermann qui exploite lentement différents types d'habitats au cours de l'année. Un suivi trop court risque de ne prendre en compte qu'une petite partie du domaine vital et, par exemple, de négliger les sites de reproduction. À ce jour, peu d'études de radio-tracking des Tortues d'Hermann ont été publiées (<10 depuis 2000); la plupart sont basées sur de faibles effectifs et des périodes assez courtes (Tableau 1). Ces suivis ne peuvent pas prendre en compte la variabilité interindividuelle, saisonnière ou interannuelle et risquent de fournir une vision 


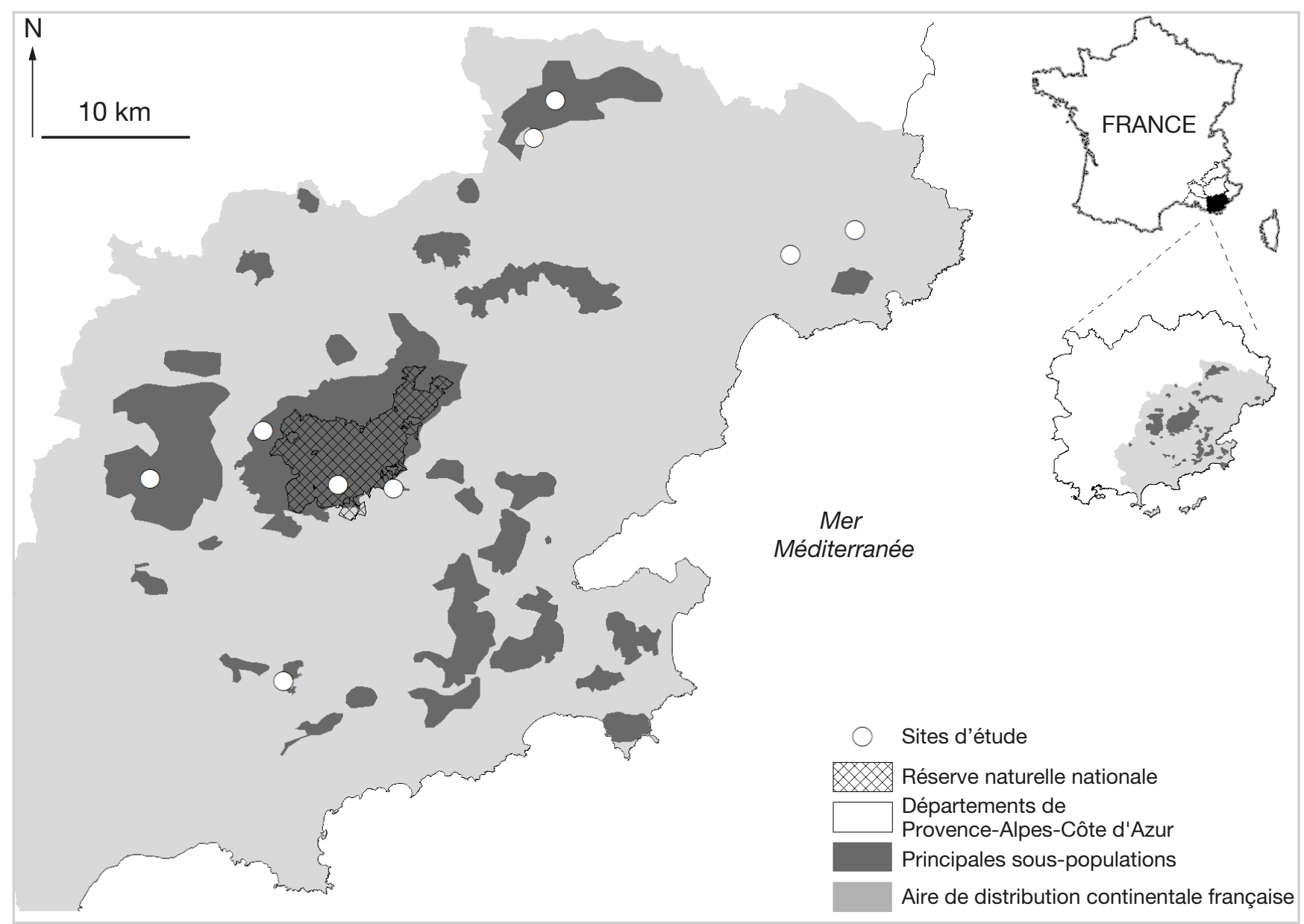

FIG. 1. - Localisation des neuf sites étudiés sur l'aire de distribution de la Tortue d'Hermann, Testudo hermanni Gmelin, 1789 en France continentale.

limitée des aires et des habitats qu’il serait nécessaire de protéger pour assurer le maintien des populations.

Afin de pallier ces carences, nous avons mis en place des suivis de Tortues d'Hermann par radio-tracking dans le Var durant plusieurs années et sur un grand nombre d'individus. Le premier objectif était de déterminer à partir de quelle intensité et durée de suivi le domaine vital d'un individu pouvait être correctement caractérisé. Le second objectif était d'évaluer la variabilité interindividuelle. Entre 2010 et 2017, plus de 160 individus ont été suivis de façon quasi quotidienne durant la totalité de la période d'activité (depuis le réveil jusqu'au retour en hibernation). Quelques tortues ont été suivies durant deux années consécutives. Ces valeurs, qui n'ont pas d'équivalent à notre connaissance, offrent une excellente opportunité d'obtenir des informations essentielles pour préserver les milieux naturels et les zones protégées.

\section{MATÉRIEL ET MÉTHODES}

\section{SiTES D'ÉTUDE}

Les suivis ont été faits sur neuf sites représentatifs de la zone principale de distribution en France métropolitaine (Fig. 1), avec des densités de population qualifiées de bonnes $(1,5-4,5$ indi- vidus/ ha; Livoreil 2009; Celse et al. 2014). La répartition des sites prend en compte la diversité des habitats, c'est-à-dire des formations végétales hétérogènes créées par le relief, l'exposition et les incendies (Tableau 2). Les sites de la Pardiguière et du Bombardier offrent un habitat typique avec une topographie relativement plane, une végétation semi-ouverte de type maquis (Erica arborea L., Pistacia lentiscus L. et Cistus spp.) et de nombreux ruisseaux et vasques temporaires. Le site de Flassans présente un habitat de type mosaïque où alternent des zones ouvertes et cultivées (plaines, vignes), des zones semi-ouvertes de type garrigue (Paliurus spina-christi Mill., Cistus monspeliensis L. et C. albidus L.) et des zones plus forestières (Quercus pubescens Willd. et Q. ilex L.). Les sites de Callas, Saint-Daumas, Lambert et Castelli sont caractérisés par des terrains vallonnés avec un habitat davantage forestier (Quercus suber L., Q. pubescens et Pinus halepensis Mill.) où le maquis a tendance à se densifier (Erica arborea). Certains sites sont inclus dans le périmètre de la réserve naturelle nationale de la plaine des Maures.

\section{RADIO-TRACKING}

Sur chacun des sites, 7 à 32 tortues, soit un total de 177 sur l'ensemble des sites, ont été équipées après avoir été pesées, mesurées et marquées à l'aide de "bagues» sur le bord avant de la carapace, complétées par un code d'encoches. 
TABlEAU 2. - Moyennes et médianes des tailles des domaines vitaux annuels en hectares (Polygones Convexes Minimum [MCP] $100 \%$ ) des Tortues d'Hermann (Testudo hermanni Gmelin, 1789) suivies par télémétrie sur neuf sites distribués dans l'aire de répartition de l'espèce dans le Var. Abréviations: *, indique si l'étude révèle une différence significative (Mann-Whitney U-tests) entre les sexes $(\mathrm{N}=165)$; $\mathrm{SE}$, erreur standard.

\begin{tabular}{|c|c|c|c|c|c|c|}
\hline \multirow[b]{2}{*}{ Site } & \multirow{2}{*}{$\begin{array}{l}\text { Type de milieu } \\
\text { dominant }\end{array}$} & \multirow[b]{2}{*}{ Période } & \multirow[b]{2}{*}{ Sexe (nombre) } & \multicolumn{3}{|c|}{ Domaine Vital annuels (MCP) } \\
\hline & & & & Moyenne \pm SE & Médianes & Min-Max \\
\hline La Pardiguière & Maquis clairsemé & 2017 & $\begin{array}{l}F(9)^{\star} \\
M(10) \\
F+M\end{array}$ & $\begin{array}{l}7,85 \pm 1,19 \\
1,97 \pm 0,41 \\
4,75 \pm 0,9\end{array}$ & $\begin{array}{l}8,18 \\
1,61 \\
3\end{array}$ & $\begin{array}{l}{[2,98-13,12]} \\
{[0,72-5,08]} \\
{[0,72-13,1]}\end{array}$ \\
\hline Bombardier & Maquis clairsemé & 2015 & $\begin{array}{l}F(5) \\
M(5) \\
F+M\end{array}$ & $\begin{array}{l}4,73 \pm 1,18 \\
4,94 \pm 1,0 \\
4,84 \pm 0,73\end{array}$ & $\begin{array}{l}4,64 \\
5,5 \\
5,1\end{array}$ & $\begin{array}{l}{[2,06-4,57]} \\
{[1,78-7,64]} \\
{[1,78-7,64]}\end{array}$ \\
\hline Neuf Riaux & Maquis clairsemé & $2012-2013$ & $\begin{array}{l}F(8) \\
M(8) \\
F+M\end{array}$ & $\begin{array}{l}3,51 \pm 0,62 \\
4,78 \pm 1,71 \\
4,14 \pm 0,89\end{array}$ & $\begin{array}{l}3,66 \\
2,86 \\
3,11\end{array}$ & $\begin{array}{l}{[1,20-6,5]} \\
{[1,36-15,79]} \\
{[1,20-15,79]}\end{array}$ \\
\hline Flassans & Mosaique agricole & $2010-2011$ & $\begin{array}{l}\mathrm{F}(22) \\
\mathrm{M}(21) \\
\mathrm{F}+\mathrm{M}\end{array}$ & $\begin{array}{l}9,92 \pm 3,23 \\
6,69 \pm 1,83 \\
8,27 \pm 1,83\end{array}$ & $\begin{array}{l}4,52 \\
4,02 \\
4,26\end{array}$ & $\begin{array}{l}{[0,55-60,09]} \\
{[1,07-45,5]} \\
{[0,55-60,1]}\end{array}$ \\
\hline Callas & Maquis dense et forêt & $2010-2011$ & $\begin{array}{l}F(17)^{\star} \\
M(15) \\
F+M\end{array}$ & $\begin{array}{r}19,8 \pm 4,41 \\
5,45 \pm 1,55 \\
13,12 \pm 2,73\end{array}$ & $\begin{array}{r}14,11 \\
5,45 \\
7,68\end{array}$ & $\begin{array}{l}{[3,26-71,3]} \\
{[1,84-26,08]} \\
{[1,84-71,3]}\end{array}$ \\
\hline Castelli & Maquis dense et forêt & 2016 & $\begin{array}{l}F(5) \\
M(4) \\
F+M\end{array}$ & $\begin{array}{r}5,49 \pm 3,69 \\
8,65 \pm 5,79 \\
6,9 \pm 3,11\end{array}$ & $\begin{array}{l}1,68 \\
3,18 \\
2,3\end{array}$ & $\begin{array}{l}{[1,62-20,25]} \\
{[2,25-25,9]} \\
{[1,62-25,99]}\end{array}$ \\
\hline Lambert & Maquis dense et forêt & 2012 & $\begin{array}{l}F(8)^{*} \\
M(6) \\
F+M\end{array}$ & $\begin{array}{c}26,98 \pm 10,53 \\
3,51 \pm 1,14 \\
16,92 \pm 6,68\end{array}$ & $\begin{array}{r}15,47 \\
3,06 \\
8,33\end{array}$ & $\begin{array}{l}{[3,70-94,9]} \\
{[1,03-8,49]} \\
{[1,03-94]}\end{array}$ \\
\hline Pennafort & Maquis dense et forêt & 2017 & $\begin{array}{l}F(5) \\
M(2) \\
F+M\end{array}$ & $\begin{array}{r}13,73 \pm 5,16 \\
6,85 \pm 0,56 \\
11,76 \pm 3,78\end{array}$ & $\begin{array}{l}7,17 \\
6,85 \\
7,17\end{array}$ & $\begin{array}{l}{[3,91-30,84]} \\
{[6,29-7,41]} \\
{[3,91-30,84]}\end{array}$ \\
\hline Saint-Daumas & Maquis dense et forêt & $2012-2013$ & $\begin{array}{l}F(14)^{\star} \\
M(11) \\
F+M\end{array}$ & $\begin{array}{r}16,03 \pm 4,08 \\
5,7 \pm 1,21 \\
11,29 \pm 2,48\end{array}$ & $\begin{array}{l}9,01 \\
4,14 \\
6,93\end{array}$ & $\begin{array}{l}{[3,39-47,67]} \\
{[2,34-15,56]} \\
{[2,34-47,67]}\end{array}$ \\
\hline
\end{tabular}

Le détail des effectifs par site est donné dans le Tableau 2; le sexe ratio était globalement équilibré (Tableau 2). Seuls les individus de plus de 400 grammes et avec une longueur de carapace supérieure à $150 \mathrm{~mm}$ ont été équipés. Deux types d'émetteur VHF ont été utilisés (Colfax AVM Instrument Company et TW-31 1/2AA BIOTRACK). Ceuxci ont été fixés sur la partie latérale-arrière de la carapace (jonction entre les écailles marginales et costales) à l'aide d'une résine armée (Fig. 2). Cette position limite la gêne lors des déplacements, l'appareil risquant de s'accrocher à la végétation. Pour l'émetteur de type Colvax, l'antenne a été fixée autour de la carapace au niveau de la jonction entre les écailles marginales et costales. Le poids du matériel et de la résine n'a jamais dépassé $10 \%$ de la masse de l'animal, valeur recommandée chez Testudo (Lagarde et al. 2008). Les individus ont été localisés grâce à une antenne Yagi à trois brins connectée à un récepteur R410ATS (Advanced Telemetry Systems, Isanti, MN, USA), et à un GPS manuel (GARMIN eTrex ${ }^{\oplus}$, marge d'erreur de $5 \mathrm{~m}$ ).

Les individus ont été équipés entre le 10 avril et le 10 mai selon les sites et les années, puis déséquipés après leur rentrée en hibernation (du 15 octobre au 30 novembre) que l'on détermine lorsque les tortues sont enterrées pendant plusieurs jours consécutifs. Les suivis couvrent ainsi l'ensemble de la période d'activité et permettent de prendre en compte l'ensemble des habitats utilisés. Les tortues ont été localisées trois à six fois par semaine, ce qui présente l'avantage de ne pas perdre leur signal lors de grands déplacements (e.g. > $300 \mathrm{~m}$ ). La plupart des individus ont été suivis durant une saison complète, mais neuf l'ont été durant deux années consécutives.

Les populations de tortues suivies sont indépendantes les unes des autres compte tenu de la distante importante qui les séparent $(>2 \mathrm{~km})$, et surtout à cause de barrières infranchissables telles que les fleuves ou les autoroutes.

\section{DOMAINES VITAUX}

Nous avons utilisé la méthode des Polygones Convexes Minimum (MCP) (Mohr 1947) avec $100 \%$ des positions incluses. Les MCP fournissent une surface minimale limitée par les points périphériques qui ne donne donc pas d'informations sur les zones les plus fréquentées (densité des localisations). Cette technique a des avantages et des limites. Pour nos objectifs tournés vers la conservation, elle permet d'obtenir une aire précise contenant toutes les localisations sans risque d'inflation des surfaces. La simplicité de calcul et d'interprétation facilite les comparaisons (Kazmaier et al. 2002; Harless et al. 2010). Chez les reptiles, la méthode des MCP entraine moins de biais et semble plus appropriée que d'autres (Row \& Blouin Demers 2006; Knight et al. 2009). C'est la méthode principalement employée dans les études antérieures (Tableau 1). Calculées sur l'ensemble de l'année, les MCP permettent de refléter l'ensemble des zones potentiellement occupées par un individu. Celles-ci contiennent à la fois 


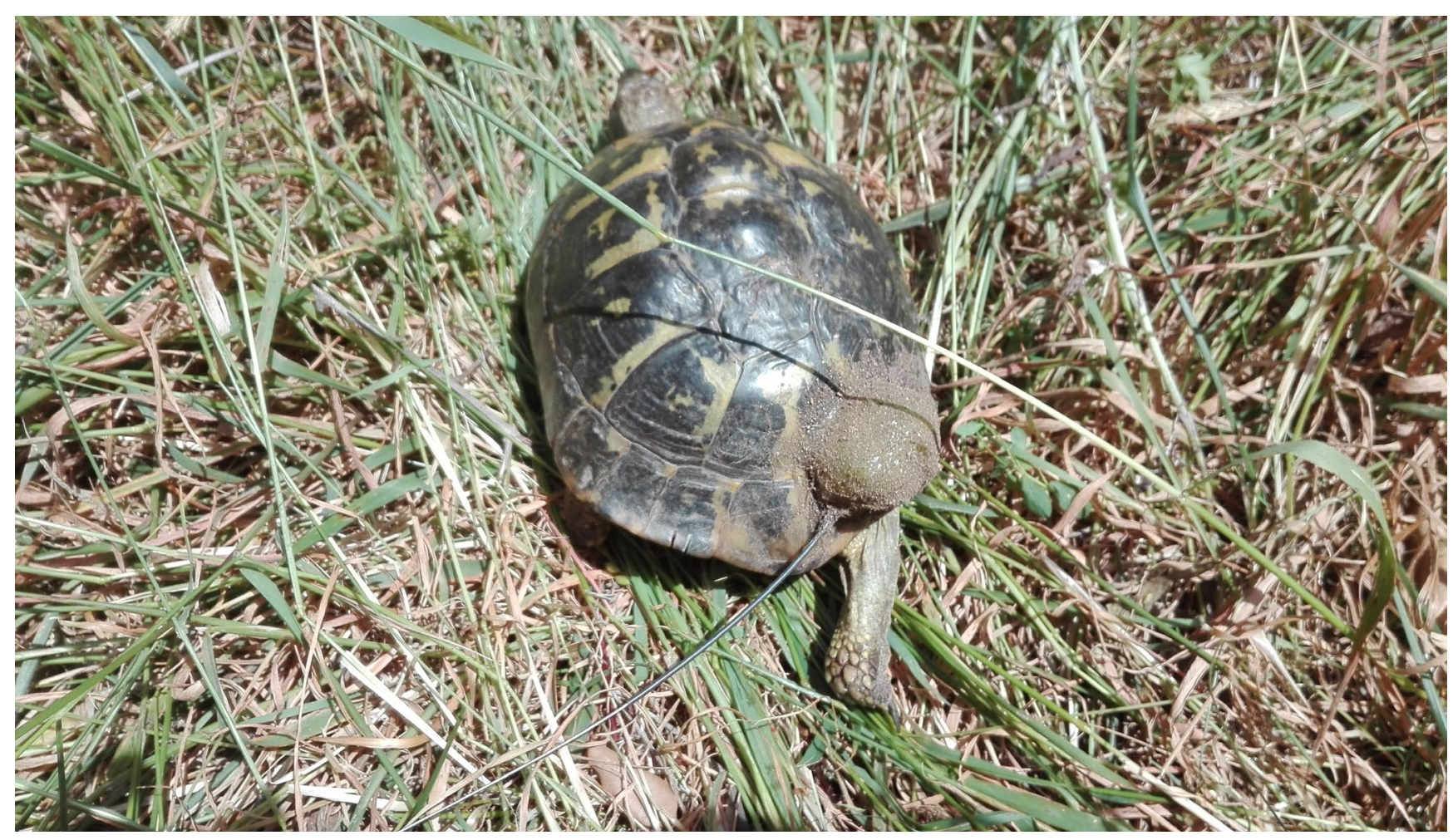

FIG. 2. - Photographie d'une Tortue d'Hermann, Testudo hermanni Gmelin, 1789 équipée d'un émetteur. Crédit photo: Élise Motteau.

les zones cœurs utilisées par les individus pour leurs besoins quotidiens (recherche de nourriture, site de thermorégulation et refuges), les zones utilisées de façon plus saisonnière tels que les sites de ponte et d'hibernation et les zones de transit utilisées entre les zones principales (Kingsburry \& Robinsons 2016). Le domaine vital MCP (DV) a été calculé pour chacune des tortues suivies avec le logiciel R ( $R$ core team 2016 [package adehabitat, fonction mcp.area]; Calenge 2006).

Pour la plupart des individus, les suivis ont effectivement pu être réalisés sur de grandes périodes. Le nombre total de localisations obtenues est 17562. Quelques cas de mortalité ( $\mathrm{N}=3$, due à la prédation) ou des défaillances techniques $(\mathrm{N}=9)$ ont légèrement réduit la taille initiale de l'échantillon $(\mathrm{N}=177)$. Nous avons pu analyser les DV annuels de 165 individus (87 femelles et 78 mâles), auxquels s'ajoutent huit DV provenant de suivis faits une année supplémentaire (trois femelles et cinq mâles suivis deux ans), ce qui donne un total de 173 DV annuels. Par simplicité, dans les analyses statistiques nous n'avons pas inclus les huit DV additionnels. Les suivis ont duré en moyenne $194 \pm 45$ jours $( \pm$ SD (erreur type), 41 à 272 jours, $N=165$ ) et le nombre moyen de localisations différentes (les tortues restent souvent au même endroit les jours défavorables) est de $99 \pm 38$ (26 à 170).

\section{ANALYSES}

L'histogramme de fréquences des DV ne suivant pas une loi de distribution normale (Shapiro-Wilk $W=0,575, \mathrm{P}<0,001$ ), une transformation Box-Cox des valeurs a été opérée (ShapiroWilk $W=0,996, P=0,923)$, ce qui a permis d'utiliser des tests paramétriques. L'évaluation de l'effort de suivi nécessaire pour obtenir une valeur stable de DV a été faite en examinant la relation entre le nombre de localisations et l'augmentation du DV. Un plafonnement de la valeur de DV suggère que l'animal ne fait plus de déplacement en dehors de l'aire décrite. Les analyses ont été effectuées à l'aide du logiciel Statistica 13.5.0.17 (Tibco Software Inc. 1984-2018).

\section{RÉSULTATS}

\section{DOMAINE VITAL}

En moyenne, le DV des Tortues d'Hermann est de 9,3 $\pm 13,0$ ha $(\mathrm{N}=165)$. La valeur médiane est de 5,2 ha, proche de la valeur modale de 4,1 ha.

RELATION ENTRE LE NOMBRE DE LOCALISATIONS ET DV Le nombre de localisations, et donc la durée du suivi, influence significativement la taille du DV $\left(\mathrm{F}_{1,163}=8,398, \mathrm{P}=0,004\right)$, mais de façon très marginale puisque plus de $95 \%$ de la variation est due à d'autres facteurs $\left(\mathrm{r}^{2}=0,043\right)$. Ce résultat est probablement en partie lié à la sélection des individus (les suivis trop courts ont été exclus), mais il est essentiellement lié aux variations interindividuelles des comportements. En effet, la Figure 3 montre que sur un sous-échantillonnage de 34 individus, le DV reste modeste et il est rapidement stabilisé avec la durée de suivi; tandis que chez d'autres, de grands déplacements induisent une augmentation forte et prolongée du DV au cours du temps. En général, au moins 
A

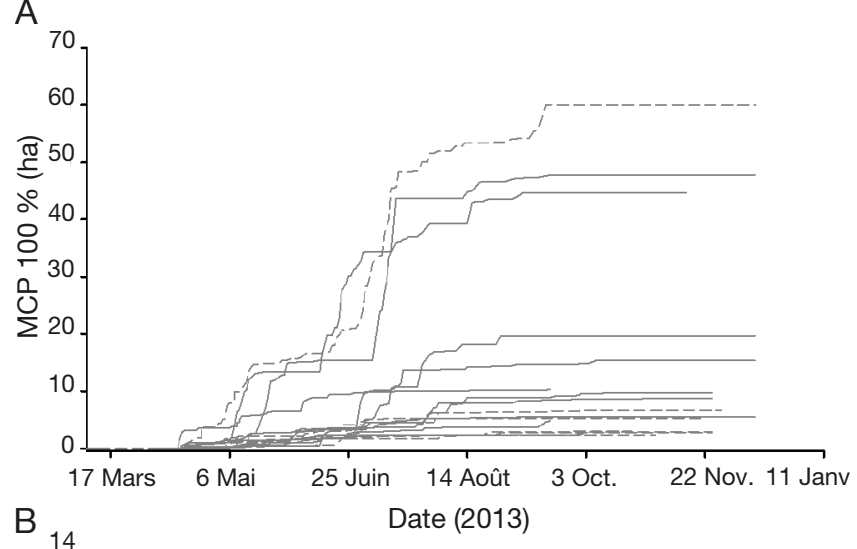

B

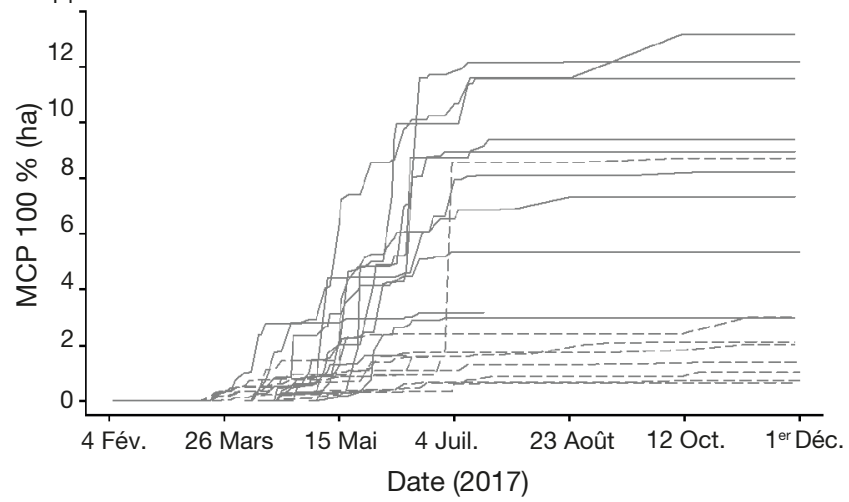

FIG. 3. - Évolution de la taille des domaines vitaux (MCP) en fonction du temps pour les individus du site de la Saint-Daumas en 2013 (A) et de la Pardiguière en 2017 (B). Les traits pleins représentent les femelles, les traits pointillés les mâles. Lorsque les tracés deviennent horizontaux, on peut considérer que le domaine vital (DV) est correctement décrit.

70 localisations (et une durée de 80 jours) ont été nécessaire pour observer la stabilisation des DV, parfois il aura fallu attendre 110 localisations (et une durée de 150 jours). Les grands déplacements se produisent à des dates variables, principalement au printemps et en été. D’une façon globale, la taille des DV augmente par paliers surtout au printemps, saison où l'activité des individus est la plus importante, puis elle se stabilise au début de la saison estivale entre le 10 juin et le 10 juillet (Fig. 3). Pour près de la moitié des individus $(\mathrm{N}=14,41 \%)$, on observe une augmentation des DV suivie d'une deuxième phase de stabilisation vers le milieu de la saison estivale $(\mathrm{N}=11,32 \%)$, avec parfois une troisième phase au début de l'automne $(\mathrm{N}=5,15 \%)$.

\section{INFLUENCE DE LA TAILLE ET DU SEXE DES TORTUES}

La taille des tortues influence positivement celle de leur DV $\left(F_{1,163}=26,743 p<0,001 ;\right.$ Fig. 4). Mais cette relation n'explique qu'une faible proportion de la variabilité des DV $\left(\mathrm{r}^{2}=0,136\right)$, plus de $85 \%$ de la variation est donc liée à d'autres facteurs. La variabilité interindividuelle très forte se traduit par des valeurs extrêmes éloignées des DV $(0,6$ ha à 95,0 ha) et un très fort coefficient de variation $(\mathrm{Cv}=140,8$, données non-transformées). Les DV inférieurs à 2 ha représentent $14 \%$ des cas, $50 \%$ sont inférieurs à 5 ha, $77 \%$ sont inférieurs à 10 ha et $23 \%$ sont supérieurs à 10 ha. La majo-

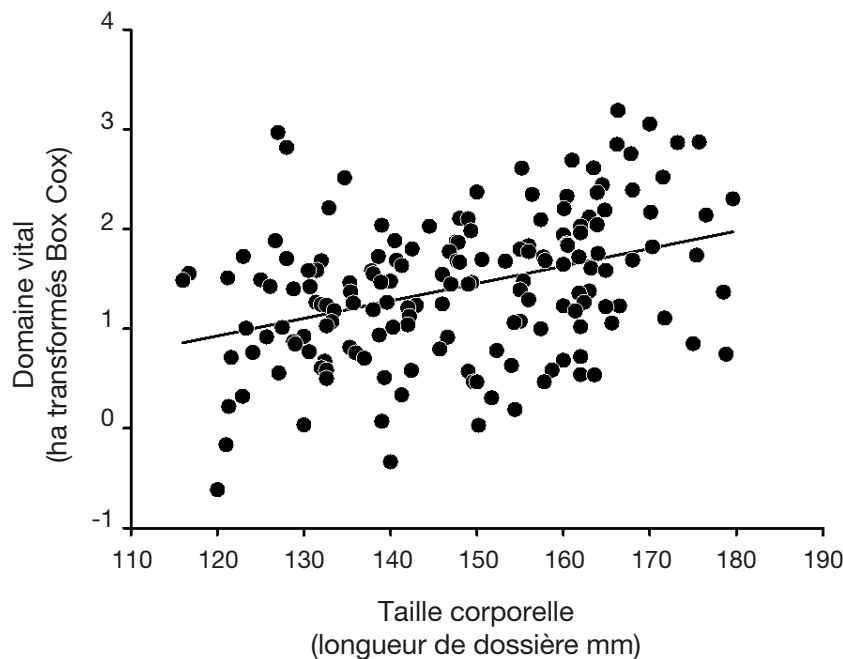

FIG. 4. - Relation entre la taille du domaine vital (DV) et la taille des Tortues d'Hermann, Testudo hermanni Gmelin, 1789.

rité des individus ayant un DV supérieur à 10 ha sont des femelles ( $82 \%, \mathrm{~N}=28 / 34)$. Cette proportion est de $92 \% \mathrm{de}$ femelles pour les DV supérieurs à 30 ha $(\mathrm{N}=11 / 12)$. Pour la majorité des sites les DV des femelles sont supérieurs à ceux des mâles (Tableau 2).

\section{DISCUSSION}

Il n'existe pas de substitut aux données de terrain précises et abondantes. Les résultats présentés dans cet article en apportent à nouveau la confirmation. Les suivis par radio-tracking menés sur la Tortue d'Hermann depuis 10 ans sur plus de 160 individus montrent que les estimations de la taille des domaines vitaux de Tortues d'Hermann que nous avons obtenues est largement supérieure à celles publiées jusqu’alors (Tableau 1).

Le premier résultat marquant est donc la grande taille des domaines vitaux annuels, avec une moyenne de 9,3 ha, un intervalle de confiance à $95 \%$ entre 7,3 ha et 11,3 ha, et une étendue considérable comprise entre 0,6 ha et 95 ha. Environ $50 \%$ des DV sont supérieurs à 5 ha, $23 \%$ sont supérieurs à 10 ha et $7 \%$ sont supérieurs à 30 ha. Ces valeurs sont non seulement loin d'être anecdotiques, elles sont aussi beaucoup plus élevées que ce qui est documenté dans la littérature, particulièrement en France où les estimations des domaines vitaux moyens n'excèdent pas 2 ha. Nos valeurs sont donc quatre à cinq fois plus élevées en moyenne, mais surtout elles changent complétement les perspectives des surfaces à prendre en compte. Ces écarts sont probablement liés au fait que les suivis antérieurs étaient brefs et faits sur un petit nombre d'animaux (Tableau 1). Par exemple, l'utilisation de fils traceurs, bien que précise à petite échelle (Longepierre et al. 2001), ne peut pas fonctionner pour décrire des DV sur de longues périodes (Fig. 3). D'ailleurs, chez la Tortue du désert (Gopherus agassizii Cooper, 1861) 60 localisations sont nécessaires pour décrire correctement le DV individuel (Rautenstrauch \& Holt 1995; Harless et al. 2010). 

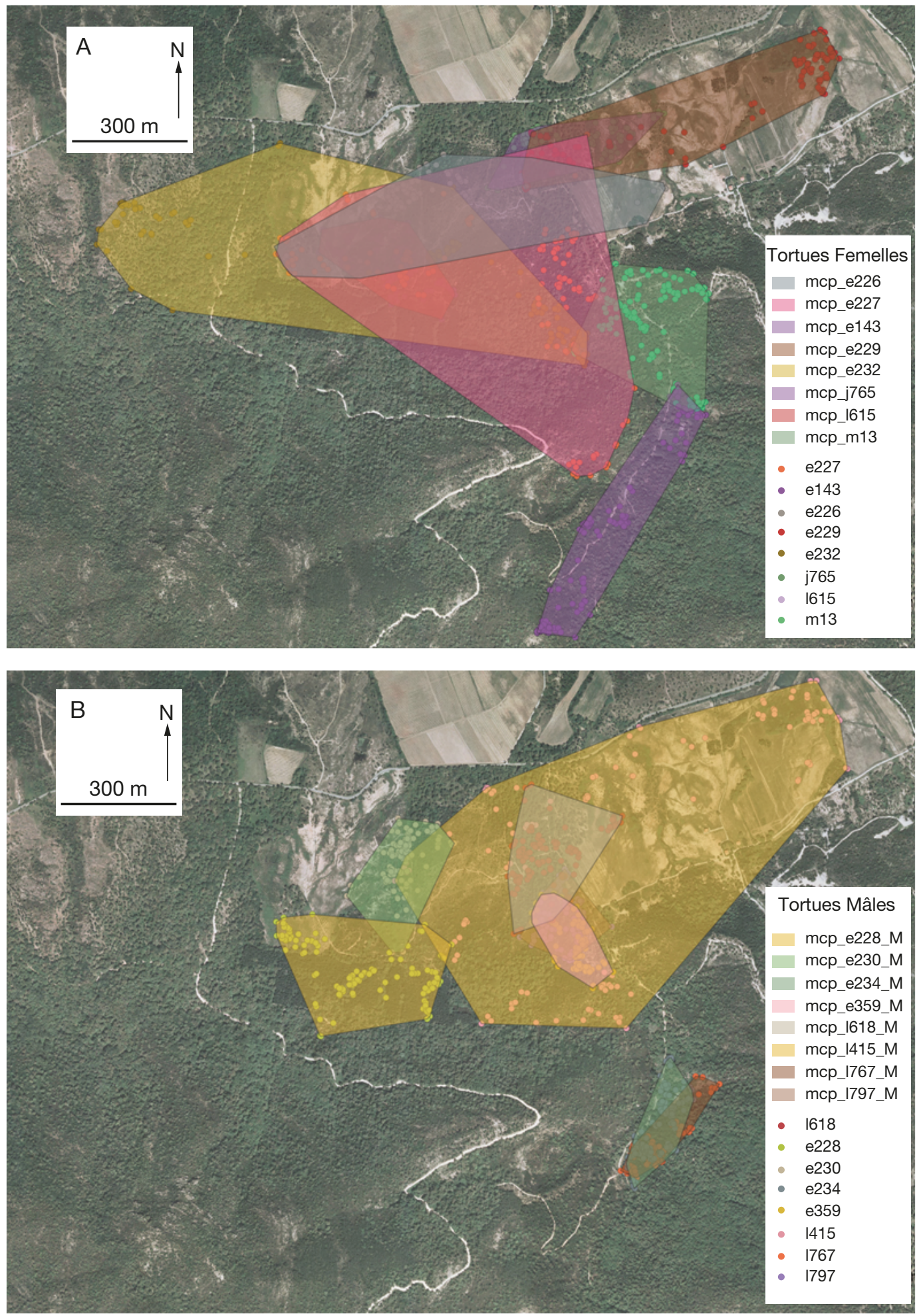

FIG. 5. - Représentation cartographique de la taille des domaines vitaux (MCP-100 \%) et des localisations pour les Tortues d'Hermann (Testudo hermanni Gmelin, 1789) femelles (A) et mâles (B) suivis à Saint-Daumas en 2013.

D'autres facteurs peuvent influencer les DV, par exemple la détérioration des habitats qui peut pousser les individus à explorer de grandes zones (Byer et al. 2017). D’une façon générale, les faibles ressources des régions désertiques s'accompagnent de grands DV (Harless et al. 2010), y compris pour les tortues du genre Testudo. Par exemple, chez la tortue des steppes (Testudo horsfieldii Grey, 1844) qui exploite des zones très arides, le DV moyen des mâles est de $24 \pm 15$ ha et celui des femelles de $57 \pm 53$ ha (Lagarde et al. 2003). En ce qui concerne la Tortue d'Hermann, des suivis faits en 
zone forestière indiquent des DV de l'ordre de 5 ha en Italie et en Espagne, une surface trois à sept fois plus importante que celles obtenues pour d'autres populations (Mazzotti et al. 2002; Casamitjana et al. 2012; Tableau 1). En milieu fermé le fourrage pourrait manquer au niveau du sol, les tortues seraient contraintes de se déplacer pour trouver leurs ressources (Longepierre et al. 2001; Mazzotti et al. 2002). Toutefois, notre étude basée sur neuf sites prend en compte des milieux différents, y compris ouverts très favorables pour l'espèce, et inclue des zones proches de celles précédemment utilisées. C'est pourquoi nous estimons que les nouvelles valeurs fournies décrivent mieux les véritables DV des Tortues d'Hermann en France métropolitaine.

Les très fortes variations interindividuelles que nous avons observées font écho à d'autres études sur la tortue d'Hermann (Luiselli et al. 2009) et sur différentes espèces de Chéloniens (e.g. Gopherus sp., Franks et al. 2011 ; Terrapene sp., Habeck et al. 2019). Nos résultats montrent à nouveau que les femelles occupent des DV en général plus grands que ceux des mâles (Lagarde et al. 2003; Bertolero et al. 2011). Les femelles entreprennent en effet de grands déplacements lors de la recherche de sites de ponte. L'exemple de la tortue «e227» peut ici illustrer ce propos (Fig. 5).

\section{IMPLICATIONS POUR LA CONSERVATION}

Chaque tortue a des habitudes et des besoins qui lui sont propres et qui changent au cours de sa vie. Toutefois, dans un cadre de conservation, il est possible d'ignorer certaines sources de variation de la taille des DV. Peu importe de savoir si le DV varie en fonction des sexes ou de l'âge puisque la viabilité d'une population nécessite la présence de toutes ces catégories d'individus. De même, de possibles variations entre les habitats ou les années ont été prises en compte grâce aux suivis à long terme sur de nombreux individus et différents sites.

En pratique, compte tenu de la forte pression anthropique qui pèse sur les habitats de l'espèce, la conservation passe par l'établissement d'espaces protégés avec des formats divers (Réserves naturelles, sites Natura 2000, Arrêté préfectoral de Protection de Biotope, etc.), ainsi que par le maintien ou le développement des continuités écologiques favorables aux déplacements (Celse et al. 2018). Le principe de précaution implique de considérer la valeur maximale des DV, valeur qui atteint régulièrement 20 ha (10\% des individus) et assez souvent deux fois plus ( 40 ha ou plus chez $5 \%$ des individus). Une superficie de 40 ha peut être considérée comme optimale; elle concerne tous les individus y compris ceux avec de plus petits DV. En effet, les petits DV sont parfois inclus dans les plus grands, pour l'essentiel les DV individuels se chevauchent (Fig. 5), mais surtout les DV individuels peuvent varier (e.g. grand DV des femelles reproductrices). Une surface de 40 ha apparaît donc comme minimale et souhaitable pour définir des espaces protégés en faveur de la conservation de la Tortue d'Hermann. En somme, de petites surfaces sont probablement limitantes pour le maintien des populations. Le cas particulier de populations restreintes à des petits refuges ne doit pas guider la politique générale.

\section{CONCLUSION}

La cible optimale à atteindre en termes de surfaces à protéger en faveur de la Tortue d'Hermann est de plusieurs centaines d'hectares. Une erreur à éviter est de limiter les espaces protégés aux zones ou habitats les plus fréquemment utilisés (densités importantes) et donc de délaisser les zones secondaires qui les bordent, mais qui sont primordiales pour la pérennité des populations. Les sites de ponte peuvent en effet être excentrés et n'être fréquentés que de façon épisodique, de même que les zones d'alimentations estivales. En dehors des limites des espaces protégés, les individus se retrouveront exposés aux risques de mortalité dus aux franchissements de route ou à une exposition face aux prédateurs inhabituels tels que les chiens et les prélèvements illégaux (Woodroffe \& Ginsberg 1998; Kramer \& Chapman 1999). De même, délaisser les espaces peu utilisés pourrait entraver la capacité des tortues à changer de zone pour s'adapter aux changements environnementaux et compromettre la connectivité entre les populations. En somme, les espaces de protection doivent être probablement les plus étendus possibles, ce qui rend leur création difficile, mais cela renforcerait également la valeur de la Tortue d'Hermann en tant qu'espèce parapluie (Longepierre et al. 2001).

\section{Remerciements}

Nous remercions tout particulièrement les nombreux stagiaires, services civiques et bénévoles sans qui les données n'auraient pu être récoltées: Mathieu Ausanneau, Maxime Barbier, Adèle de Baudoin, Manon Batista, Coralie Borreil, Stacy Beaugier, Elise Beck, Gopal Billy, Clément Blin, Sarah Boilet, Solène Briard, Gilles Bougere, Roxane Bron, Delphine Busson, Kathy Cano, Laureline Chabran, Corinne Chaize, Ludovic Collet, Vincent Danias, Fran De Coster, Axelle Denis, Morgan Deviras, MariePauline Dessert, Christabelle Duhamel, Aurélia Dubois, Alice Ekori, Isabelle Epaillard, Thomas Etien, Matthieu Falher, Thomas Ferrari, Kévin Fourniere, Caroline Gayet, Baptiste Genolet, Antoine Gilet, Margot Gomez, Florent Girard, Camille Gravier, Eddy Ghetty, Quentin Guillaury, Fanny Gossens, Anna Guinet, Marie Jehanno, Laureline Jean, Mathieu Jegu, Julie Jourdan, Thibault Lafon, Oriane Lepeigneul, Kévin Lespinasse, Paul Lallement Céline Luciano, Gaelle Marday, Vincent Mariani, Cécile Maurand, Elise Motteau, Gaelle Micheli, Amaury Peyrot, Marie PierreJean, Quentin Reboulet, Yolan Richard, Mattias Pires, François Pierrard, Laure Servant, Adélaïde Sibeaux, Juliette Tardivy, Olivier Trepos , Marjorie Tortel, Océane Thusy, Manon Valat, Alexis Veldeman, Emmanuelle Vignes, Antoine Vignol, Julie Vingere. Nous remercions également les deux rapporteurs: Philippe Gourdain et Marc Cheylan pour leur contribution à l'amélioration de ce manuscrit. Cette étude a en partie bénéficiée du soutien financier du programme européen Life+ «Tortue d'Hermann» (2010-2014).

\section{RÉFÉRENCES}

Berardo F., Carranza M. L., Frate L., Stanisci A. \& Loy A. 2015. - Seasonal habitat preference by the flagship species 
Testudo hermanni: implications for the conservation of coastal dunes. Comptes Rendus Biologie 338 (5): 343-350. https://doi. org/10.1016/j.crvi.2015.03.002

BerTOlero A. 2002. - Biología de la tortuga mediterránea Testudo hermanni aplicada a su conservación. M. Sc. thesis, Universidad de Barcelona, Barcelona, 225 p.

Bertolero A., Cheylan M., Hailey A., Livoreil B. \& WillemSEN R. E. 2011. - Hermann's tortoise. Conservation biology of freshwater turtles and tortoises: a compilation project of the IUCN/SSC Tortoise and Freshwater Turtle Specialist Group. Chelonian Research Monograph 5: 059-1. https://doi.org/10.3854/ crm.5.059.hermanni.v1.2011

Börger L., Franconi N., De Michele G., Gantz A., Meschi F., MANICA A. \& COULSON T. 2006. - Effects of sampling regime on the mean and variance of home range size estimates. Journal of Animal Ecology 75 (6): 1393-1405. https://doi.org/10.1111/ j.1365-2656.2006.01164.x

Bossuto P., Giacoma C., Rolando A. \& Balletto E. 2000. Caratteristiche delle aree familiari in una popolzione di Testudo hermanni Gmelin del Parco Nazionale della Maremma (GR), in Giacoma C. (éd.), Atti/Congresso Societas Herpethologica Italica. Museo Regionale Scienze Naturali, Torino: 543-551.

BURT W. H. 1943. - Territoriality and home range concepts as applied to Mammals. Journal of Mammalogy 24: 346-352. https:// doi.org/10.2307/1374834

Byer N. W., Smith S. A. \& Seigel R. A. 2017. — Effects of site, year, and estimator choice on home ranges of Bog Turtles (Glyptemys mublenbergii) in Maryland. Journal of Herpetology 51 (1): 68-72. https://doi.org/10.1670/15-150

CALENGE C. 2006. — The package "adehabitat» for the R software: a tool for the analysis of space and habitat use by animals. Ecological Modelling 197: 516-519. https://doi.org/10.1016/j. ecolmodel.2006.03.017

Calzolai R. \& Chelazzi G. 1991. - Habitat use in a central Italy population of Testudo hermanni Gmelin (Reptilia Testudinidae). Ethology ecology \& evolution 3 (2): 153-166. https://doi.org/10. 1080/08927014.1991.9525381

Castellón T. D., Rothermel B. B. \& Bauder J. M. 2018. Gopher Tortoise Burrow use, home range, seasonality, and habitat fidelity in scrub and mesic flatwoods of southern Florida. Herpetologica 74: 8-21. https://doi.org/10.1655/HerpetologicaD-17-00030.1

Casamitjana M., loaiza J. C., Simon N. \& Frigola P. 2012. Aspectos ecológicos y efectos del manejo forestal en una población de Tortuga mediterránea (Testudo hermanni hermanni) en Cataluña (españa). Basic and applied Herpetology 26: 73-86. https:// doi.org/10.11160/bah.11018

Celse J., Catard A., Caron S., Ballouard J.-M., Roux A., ChEYlan M. \& BOSC V. 2018. - Plan national d'actions "Tortue d'Hermann" 2018-2027. Conservatoire d'Espaces Naturels de Provence-Alpes-Côte d'Azur, Le Luc, 120 p.

Celse J., Catard A., Caron S., Ballouard J.-M., Gagno S., Jarde N., Cheylan M., Astruc G., Croquet V. \& Bosc M. 2014. - Guide de gestion des populations et des habitats de la Tortue d'Hermann. Arpe PACA, Aix-en-Provence, 210 p.

Chelazzi G. \& Francisci F. 1979. - Movement patterns and homing behavior of Testudo hermanni Gmelin (Reptiliates Tudinidae). Monitore zoologico italiano 13: 105-127.

Chelazzi G. \& Calzolai R. 1986. - Thermal benefits from familiarity with the environment in a reptile. Oecologia 68: $557-$ 558. https://doi.org/10.1007/BF00378771

Cheylan M. 1984. - The true status and future of Hermann's Tortoise Testudo hermanni Robertmertensi Wermuth 1952 in Western Europe. Amphibia-Reptilia 5: 17-26. https://doi. org/10.1163/156853884X00048

Cheylan M., Couturier T. \& Astruc G. 2013. — Où en est la protection de la Tortue d'Hermann en France? Revue scientifique Bourgogne-Nature 17: 156-161.
Cheylan M., Catard A., Livoreil B. \& Bosc V. 2009. - Plan national d'actions en faveur de la Tortue d'Hermann, Testudo hermanni hermanni. DREAL PACA, Marseille, 137 p.

CORBETT K. 1989. - Conservation of European Reptiles and Amphibians. Helm, London, 288 p.

Couturier T., Tillion-Lacazale L., Besnard A., Astruc G. \& Cheylan M. 2014. - Déclin d'une population de Tortue d'Hermann (Testudo hermanni hermanni Gmelin, 1789) en milieu forestier. Effets combinés de plusieurs facteurs anthropiques. Revue d'écologie (Terre et vie) 69 (2): 142-150.

Devillard S., Aubineau J., Berger F., LéOnard Y., RoObrouck A. \& MARCHANDEAU S. 2008. - Home range of the european rabbit (Oryctolagus cuniculus) in three contrasting French populations. Mammalian Biology 73: 128-137. https://doi.org/10.1016/j. mambio.2007.01.003

Di Franco A., Plass-Johnson J. G., Di Lorenzo M., Meola B., Claudet J., Gaines S. D., García-Charton J. A., Giakoumi S., Grorud-Colvert K., Werner Hackradt C. \& Micheli F. 2018. - Linking home ranges to protected area size: the case study of the Mediterranean Sea. Biological Conservation 221: 175-181. https://doi.org/10.1016/j. biocon.2018.03.012

Franks B. R., Avery H. W. \& Spotila J. R. 2011. — Home range and movement of desert tortoises Gopherus agassizii in the Mojave Desert of California, USA. Endangered species research 13 (3): 191-201. https://doi.org/10.3354/esr00313

Gese E., Rongstad O. J. \& Mytton W. R. 1988. - Home range and habitat use of coyotes in southeastern Colorado. Journal of wildlife management 52 (4): 640-646. https://doi. org/10.2307/3800923

GuisAn A. \& Hofer U. 2003. - Predicting reptile distributions at the mesoscale: relation to climate and topography. Journal of Biogeography 30 (8): 1233-1243. https://doi.org/10.1046/j.13652699.2003.00914.x

Habeck C. W., Figueras M. P., Deo J. E. \& Burke R. L. 2019. A surfeit of studies: what have we learned from all the Box Turtle (Terrapene carolina and T. ornata) home range studies? Diversity 11(5): 68. https://doi.org/10.3390/d11050068

Hailey A. 1989. - How far do animals move ? Routine movements in a tortoise. Canadian Journal of Zoology 67 (1): 208-215. https://doi.org/10.1139/z89-028

Hailey A. \& Coulson I. M. 1996. - Differential scaling of home-range area to daily movement distance in two African tortoises. Canadian Journal of Zoology 74 (1): 97-102. https:// doi.org/10.1139/z96-013

Harless M. L., Walde A. D., Delaney D. K., Pater L. L. \& HAYES W. K. 2010. — Sampling considerations for improving home range estimates of desert tortoises: effects of estimator, sampling regime, and sex. Herpetological Conservation Biology 5 (3): 374-387.

HuOT-Daubremont C. 1996. - Contribution à l'étude écophysiologique de différents aspects du cycle annuel de la Tortue d'Hermann (Testudo hermanni hermanni) dans le massif des Maures (Var). Thèse, Tours, $181 \mathrm{p}$.

Hinam H. I. \& Clair C. C. S. 2008. - High levels of habitat loss and fragmentation limit reproductive success by reducing home range size and provisioning rates of northern saw-whet owls. Biological Conservation 141 (2): 524-535. https://doi.org/10.1016/j biocon.2007.11.011

Kazmaier R. R., Hellgren E. C. \& Ruthyen D. C. 2002. Home range and dispersal of Texas tortoises, Gopherus berlandieri, in a managed thorn scrub ecosystem. Chelonian Conservation Biology 4: 488-496.

Kingsbury B. A. \& Robinson N. J. 2016. - Movement patterns and telemetry, in Kenneth Dodd C. (éd.), Reptile Ecology and Conservation: a Handbook of Techniques. Oxford University Press., Oxford: 110-121. https://doi.org/10.1093/acprof: oso/9780198726135.001.0001 
Knight C. M., Kenward R. E., Gozlan R. E., Hodder K. H., Walls S. S. \& LuCAS M. C. 2009. - Home-range estimation within complex restricted environments: importance of method selection in detecting seasonal change. Wildlife Research 36 (3): 213-22. https://doi.org/10.1071/WR08032

Kramer D. L. \& ChAPMAN M. R. 1999. - Implications of fish home range size and relocation for marine reserve function. Environmenatal Biology of Fish 55 (1-2): 65-79. https://doi. org/10.1023/A:1007481206399

Lagarde F., Bonnet X., Henen B., Legrand A., Corbin J., Nagy K. \& Naulleau G. 2003. - Sex divergence in space utilisation in the steppe tortoise (Testudo horsfieldi). Canadian Journal of Zoology 81 (3): 380-387.

Lagarde F., Guillon N., Dubroca L., Bonnet X., Kaddour K. B., Slimani T. \& El Mouden E. H. 2008. - Slowness and acceleration: a new method to quantify the activity budget of chelonians. Animal Behaviour 75: 319-329.

LiVOREIL B. 2009. - Distribution of the endangered Hermann's tortoise Testudo hermanni hermanni in Var, France, and recommendations for its conservation. Oryx 43: 299-305. https://doi. org/10.1017/S0030605307000841

Luiselli L., Rugiero L., Celleti S., Papi R., Gracceva G., Stacchiotti M., Mancini F., Berretta G., Berretta L., Bombara G., Fiaschetti R., Lucioli E., Trionfetti M. G. \& UNGARO A. 2009. - Autumnal home range in radio-tracked tortoises (Testudo hermanni) from a semi-arid Mediterranean environment. Revue d'écologie (Terre et vie) 64: 73-78.

Longepierre S., Hailey A. \& Grenot C. 2001. — Home range area in the tortoise Testudo hermanni in relation to habitat complexity: implications for conservation of biodiversity. Biodiversity and Conservation 10 (7): 1131-1140. https://doi. org/10.1023/A:1016611030406

McMaster M. K. \& Downs C. T. 2009. - Home range and daily movement of Leopard Tortoises (Stigmochelys pardalis) in the Nama-Karoo, South Africa. Journal of Herpetology 43 (4): 561-570.

Maxwell S. M., Breed G. A., Nickel B. A., Makanga-Bahouna J., Pemo-Makaya E., Parnell R. J., Formia A., Ngouessono S., Godley B. J., Costa D. P. Witt M. J. \& Coyne M.S. 2011. Using satellite tracking to optimize protection of long-lived marine species: olive ridley sea turtle conservation in Central Africa. PloS one 6 (5): 19905. https://doi.org/10.1371/journal.pone.0019905

MazZOtTi S., Pisapia A. \& Fasola M. 2002. - Activity and home range of Testudo hermanni in northern Italy. Amphibia-reptilia 23 (3): 305-312. https://doi.org/10.1163/15685380260449180

MoHr C. O. 1947. - Table of equivalent populations of North American small mammals. American Midland Naturalist 37 (1): 223-249. https://doi.org/10.2307/2421652

Powell R. A. \& Mitchell M. S. 2012. - What is a home range? Journal of Mammalogy 93 (4): 948-958. https://doi. org/10.1644/11-MAMM-S-177.1

Rautenstrauch K. R. \& Holt E. A. 1995. - Selecting an appropriate method for calculating Desert Tortoise home range size and location, in FLETCHER-JONES A. (éd.), Proceedings of the 1994 Symposium of the Desert Tortoise Council. Desert Tortoise Council, Inc, California, San Bernardino: 172-173.

R CORE TEAM 2016. - $R$ : a language and environment for statistical computing. $\mathrm{R}$ foundation for statistical computing, Vienna. https://www.r-project.org/

ROZYLOWICZ L. \& POPESCU V. D. 2013. - Habitat selection and movement ecology of eastern Hermann's tortoises in a rural Romanian landscape. European Journal of wildlife Research 59 (1): 47-55. https://doi.org/10.1007/s10344-012-0646-y

Row J. R. \& Blouin-Demers G. 2006. - Kernels are not accurate estimators of home-range size for herpetofauna. Copeia 2006 (4): 797-802. https://doi.org/10.1643/00458511(2006)6[797:KANAEO]2.0.CO;2

Stojadinovic D., Milosevic D., Sretic K., CvetKovic M., Jovanovic T., Jovanovic B. \& Isailovic J. C. 2017. — Activity patterns and habitat preference of eastern Hermann's tortoise (Testudo hermanni boettgeri) in Serbia. Turkish Journal of Zoology 41 (6): 1036-1044. https://doi.org/10.3906/zoo-1702-21

Swingland I. R., Stubbs D., Newdick M. \& Worton B. 1986. Movement patterns in Testudo hermanni and implications for management, in RôCEK Z. (éd.), Studies in herpetology. Charles university, Prague: 573-578.

TOMASETTI G. 1997. - Indagini morfometriche ed ecologiche su una popolazione di Testudo hermanni hermanni Gmelin dei Monti Nebrodi (Sicilia). Tesi di Dottorato, Università Studi, Catania, $184 \mathrm{p}$.

Türkozan O., Karaman S., Yilmaz C. \& Ülger C. 2019. Daily movements and home range of Eastern Hermann's Tortoise, Testudo hermanni boettgeri (Reptilia: Testudines). Zoology in the Middle East 65 (1): 28-34. https://doi.org/10.1080/093 97140.2018 .1552292

UICN FRANCE, MNHN \& SHF 2015. - La Liste rouge des espèces menacées en France - Chapitre Reptiles et Amphibiens de France métropolitaine. UICN France, MNHN, SHF, Paris, 12 p.

Vilardell-Bartino A., Capalleras X., Budó J., Bosch R. \& PONS P. 2015. - Knowledge of habitat preferences applied to habitat management: the case of an endangered tortoise population. Amphibia-reptilia 36 (1): 13-25. https://doi. org/10.1163/15685381-00002971

White G. W. \& GARROT R. A. 1990. - Analysis of wildlife radiotracking data. Academic press, San Diego, 383 p.

WoOdRofFe R. \& GinsBERG J. R. 1998. - Edge effects and the extinction of populations inside protected areas. Science 280 (5372): 2126-2128. https://doi.org/10.1126/science.280.5372.2126

Soumis le 15 novembre 2019; accepté le 27 janvier 2020; publié le 9 septembre 2020 